inter Cattolicos, qui prætextu zeli et pietatis statum politicum ubique perturbant, et ad democratiam omnia trahunt ut ipsi interim omnia regant.

Responsum $\mathrm{S}^{\mathrm{mi}}$.

Videbimus quid de his dicat orator Christianissimi, et faciemus quicquid Catholicis prodesse iudicabimus sine religionis aut sedis huius preiudicio.

21. Vna Nota per il p're Holto e tali confidenti amici à gli quali lui trouera buono de communicarla. ${ }^{\mathrm{a}}$

Le cause principali di questo mio viaggio sono de assettarsi con sua Santità et il P're generale tutti tali punti che si uederanno necessarij per il sostento degli seminarij de Spagna, Fiandra et Italia, et de gli missionarjj de la società in Inghilt ${ }^{\mathrm{a}}$, et però tutto quello che si presenterà a uoi intorno à quelli punti, cioè delle facultì, gouerno, priuilegij, e sostento, ò cose simili, io ui pregho et gli altri amici, di auuisarmi con tutta la breuità possibile, perche l' intentione mia 8 de procurare che io no mene resti in Italia seno il manco che sia possibile io hò promesso in Spagna, et per diuersi ragioni serà molto necessario.

S' io posso ancora far' qualche opera bona nel co'porsi et accordarsi le con'uersie del Sem ${ }^{\text {rio }}$ Inglese Romano, et delle differenze trà gl' altri della nat ${ }^{\text {ne }}$ n'ra altroue, farò il meglio che posso, al manco spero di far' intendere à S.S. $S^{\text {ta }}$ et all' altre persone principali, il fundamento 54, 1. 144 . et le uere cause di queste controuersie.

Intorno alle cose del stato $d^{\prime}$ Inghilta io intendo de mostrare al Papa come se ne stanno, e quanto sia necessario che sua $\mathbf{S}^{\text {ta }}$ si ne

- Tierney printed another abstract of this letter, dated correctly March 15, from the Italian in Parsons' own handwriting; and Plowden published an English translation in his Berington's Panzani, p. 350. Parsons himself printed a great part of it in English in his Manifestation, prudently omitting here, however, the passage about the Infanta. Tierney remarks that in the following July Parsons wrote to Juan d'Idiaquez that he had had an audience of the Pope, who "appeared as warm in the cause of the Infanta as could be desired" (iii. lvii-lix).

VOL. II. 
pensa da uero di quel nego con breuità affin che dopò la morté della Rega d' Inghilt non sene uenga à mani, peggiori gli pericoli, o danni inevitabili che seguiteranno si qualche si uoglia Principo heretico preuale: Che gli Cattolici Inglesi desiderano solamente un Catt $^{\text {co }}$ Rè senza rispetto che sia Inglese, Scozzeze, ò Spagnolo; il che in questo dipende principalmente di sua Santità, che il Padre Personio no è inimico del Rè di Scotia ò agente per il Rè di Spagna come alcuni hanno informati, mostrando per il primo gli buoni officij che il P're Personio hà fatto per il Rè di Scotis per molti anni mentre che si era speranza che diuentasse Cattholico.

Et per il secondo mostrando per il testimonio del Nuntio de Madrid (il quale hà scritto efficacemente à questo fine) che il P. Personio ha tuttauia persuaso al Rè, et à gli ministri suoi, che nò conuiené che sua $\mathrm{Ma}^{\mathrm{ta}}$ pretende Inghilt per lui, et che il P. Personio hà impetrato del Rè di Spagna una promessa absoluta de cio fare intorno à quel punto, il Nuntio hà visto gli discorsi, et è stato fatto consapeuole delle Conferenze et ragionamenti che il P. Personio ha fatto de giorno in giorno à quel fine.

In fine questa deue ser la conelusione che la sola strada è, che S. $S^{t a ̀ ~} s^{\prime}$ accordasse con il Rè di Spagna de qualche compositione ragioneuole per qualche persona che serà capabile, e che starà bene, 54, f. 144b. per Sua Sanc ${ }^{\text {tà }}$, sua Maestà Cattca, Inglesi, e Scozzezi, il Rè di - Nauarra, denemarca e tutti gli altri. Ma che sarà questa persona ò persone l' intentione del Padre Personio è di lasciar' à pensare à S. $S^{\text {ta }}$ e de rompere la testa sua per qualche tempo.

Però al parere mio no sene troua altra compositione più profitabile, probabile, et factibile, che la Infanta con il Principe Cardinale," mà si noi altri gli buoni amici nostri siate di un' altro parere, $\theta$ possiate proporne gli mezzi, di gracia mettete gli per iscritto, perche mi rallegrerò de sentirne et accommodarmi à uoi altri ancora; perche in quest' altro uoglio andar pianpiano fin tanto che habbia uostra risposta, et ricordarsi che in questo non si hà da mirare solamente quello che sia conforme à i nostri desiderij et appetiti, "Parsons' own copy underlines ' signora infanta maritata al principe cardinale.' 
mà quelle trè conditioni inanzi specificate de profitto, probabilità, et factibilita, tanto de preualersi e guadagnar, quanto di defendere, sentare, e continuarsi dipoi, et questo è tutto quello che in questo punto io posso dire, et anzi basciando di cuore gli mani a tutti, ui dico adio, desiderando in questi negotij tutta la secretezza possibile come nedete che sia necessaria: Il n'ro Sor Jesus resti sempre con voi altri. di Genoua à gli 15. di maggio [sic] 1597.

Vostro sempre, la mano del quale conoscete.

Has esse P'ris Personij literas dum ex Hispania Romam nenit et [jam eas] propria sua manu conscriptas habemus in Vrbe tres uiros fidedignos qui confirmabunt tanquam oculati testes.

54, t. 145.

$2^{\circ}$. Habemus Prototypon in Gallijs manu sua propria conscriptum.

$3^{\circ}$. Argumento sunt illum hoc animo Romam uenisse anno 1597. et hec in itinere scripsisse, liber quem de Successione scripsit cuins conclusio eadem est quæ harum literarnm pro Infantæ cum Cardinale [matrimonio]; subscriptiones quas ab alumnis collegiorum in hunc finem exegit; instructiones quas sacerdotibus in Angliam missis sibique confidentibus de Infantæe titulo promonenido dedit, vnde merito suspecta possunt esse omnia quæ de Archipresb'ro promouendo et defendendo tam acritèr hactenus egit tanquam qui abuti noluerit Pont ${ }^{\text {cis }}$ pijssima intentione ad factionem hispanicam in Anglia stabiliendam.

In his literis Patris Personij multa sunt notatu digna; illud nero precipuè examinandum censemus: quot olim hanc esse uiam statuit ad conuertendam Angliam, nimirum ut sua Sanc ${ }^{\text {tas }}$ cum Rege Cattco conueniat de compositione aliqua facienda cum successore aliquo idoneo qui æque gratus uideatur Pontifici, Regi Cattolico, Anglis et Scotis Catholicis, Regi Nauarræ, Danemarcæ, et reliquis omnibus.

Hanc autem personam esse Infantam cum Cardinale tàm hìc quàm in libro suo de Successione concludit proptèr utilitatem, probabilitatem et factibilitatem, ut ipsius nerbis utamur. 
Et primo notandum est Suæ Sanctitati magnam factam esse iniuriam quod biennio post absolutionem datam Regi Christmo dedignatur nomen Regis Galliæ, sed Nauarræ solum in contemp54, f. 145b. tum, ut prius solebat, quod ipsum in Anglia alius Jesuita, qui nihil preterquamquod Patri Personio placuerit loqui audet, apertius promulgauit, hereticum eum et peiorem heretico appellans, Papamque in eius absolutione male fuisse informatum et a Theologo suo delusum affirmans, cuins rei testes habemus sacerdotes suos; et quorsum hæc tendant et unde motus hæc dixerit relinquimus judicio Ill mae $\mathrm{D} . \mathrm{V}$.

\section{De Vtilitate.}

Mirum est Infantam et Cardinalem qui in Belgia à Regni Prouincijs et Principibus aluntur, qui sumptus belli non possunt sustinere nisi continuis exactionibus, impositionibus et contributionibus populi, quomodo possint Regno Angliæ tantum afferre commoditatis ut utilius nihil excogitari possit.

\section{De Probabilitate.}

Non est probabile Infantam quæ patrimonium suum in Belgia uix potest à turba quadam rebelli et factiosa subditorum suorum defendere posse illud regnum alienum subiugare, tot externis, et internis competitoribus emulis et inimicis undique imminentibus.

De Factibilitate.

Nisi externo et alieno milite rem agat Infanta in Anglia, nisi 54, f. 146. post prostratos inimicos, non est quod de Catholicorum presumat aut potentia aut beneuolentia qui nec adeo sunt potentes, ut solet male informare Pater Personius, nec tàm benê affecti in Hispanos ut uelint pro eis periclitari.

Sunt enim in Anglia professi notique ut Catholici 30,000. plus minus, et ex eis pars maior feminarum, puerorum, seruulorum, pauci admodum primarij viri, ex nobilitate nix duo paria, et hi non omnes in Hispaniam affecti.

Solet autem Pater Personius fortassis numerum Catholicorum ad $10,000[100,000$ ? $]$ extendere, affectionem, et zelum in His- 
paniam predicare, suoque nutu et arbitratu regi, ut maiorem habeat cum sua sanctitate et rege Cattolico auctoritatem, fictis et simulatis literis et relationibus insinuare.

Cum in Anglia Infanta semper hereticos habebit infestissimos, et Catholicos paucos et tepidos, et à tergo Regem Scotiæ qui ius suum uindicare conabitur, et à fronte Regem Christianissimum qui nullo modo uicinitatem illam ferre persuaderi potest, et à latere Hollandos et Danos mari potentissimos, et nulli[bi] amicos aut confederatos aliquos; nisi quos pecuniæ vi ex remotissimis regionibus uocauerit, nescio quid in mentem uenerit cordatis Principibus tàm uana spe huc usque decipi, et de medio tàm impossibili tanquam de solo et unico Angliæ medicamento cogitare.

22. Vera breuisque Declaratio Status et Conditionis Catholicorum in $54,1.146 \mathrm{~b}$. Anglia ab anno Dñi 1587 vsque ad hodiernum diem.

$1^{\text {mo }}$ In tota Insula nulla est Ecclesia, nullum sacellum, locus nullus ubi Catholici aut publicè aut priuatim possint aut sacro interesse aut alia frequentare sacramenta ad salutem animarum necessaria.

$2^{\circ}$. Qui hereticorum conciones et conuenticula frequentare recusent, singulis annis 660 . aureos fisco persoluunt, quod si non sint soluendo, in carceres conijciuntur.

$3^{\circ}$. Pena Capitis est Ecclesiæ Romanæ reconciliari, pecata confiteri, à peccatis absolui.

40. Pena Capitis est sacerdotem hospitio recipere, auxilio, consilio, aut re iuuare.

50. Pena Capitis est sacerdotem, si cognoueris, illico magistratui non manifestare.

$6^{\circ}$. Nemo est alicuius notæ Catolicus quin in Custodia aliqua teneatur, hi in arctiori, illi in laxiori.

$7^{\circ}$. Nemo Catholicorum aut arma domi habere aut officio in republica frui potest. 